\title{
Síndrome de Herlyn-Werner-Wunderlich: reporte de caso y revisión de la literatura
}

\section{Herlyn-Werner-Wunderlich syndromne: case review and report of the literature}

\author{
Oscar Octalivar Gutiérrez-Montufar, $\mathbf{M D}^{1}$; Claudia Patricia Zambrano-Moncayo, MD $^{2}$; \\ Maria Camila Otálora-Gallego, $\mathrm{MD}^{3}$; Angy Lorena Meneses-Parra, $\mathrm{MD}^{4}$; Ivonne \\ Díaz-Yamal, $M^{5}$ \\ Recibido: 22 de abril del 2021/Aceptado: 8 de noviembre del 2021
}

\section{RESUMEN}

Objetivo: presentar un reporte de caso de síndrome de Herlyn Werner Wunderlich (SHWW) y hacer una revisión de la literatura para determinar los hallazgos clínicos e imagenológicos en estas pacientes.

Materiales y métodos: se presenta el caso de una mujer de 16 años que consultó, en un hospital de las fuerzas militares en Bogotá, por dolor pélvico recurrente, su diagnostico final fue SHWW. Se realizó una búsqueda sistemática de la literatura en las diferentes bases de datos, revisiones sistemáticas, cohortes, series de casos y reportes de casos en pacientes con diagnóstico de SHWW en cualquier edad, se obtuvo información sobre las características de presentación clínica, y las tecnologías diagnósticas más frecuentemente utilizadas. Se hace resumen narrativo de los hallazgos.

Resultados: se incluyeron 77 publicaciones, un total de 676 pacientes. Los síntomas más frecuen-

* Correspondencia: Oscar Octalivar Gutiérrez-Montufar. Cra 4 E 19 N 44, Popayán, Cauca (Colombia). ogutierrezm@unicauca.edu.co Descargos de responsabilidad: el contenido del presente artículo es de responsabilidad exclusiva de los autores y no compromete la opinión de la revista.

1. Residente de Ginecología y Obstetricia de la Universidad del Cauca. Popayán (Colombia)

2. Especialista en Ginecología y Obstetricia, Fundación Universitaria Sanitas, Bogotá (Colombia).

3. Especialista en Ginecología y Obstetricia de la Universidad Militar Nueva Granada, Bogotá (Colombia).

4. Residente de Ginecología y Obstetricia de la Universidad Militar Nueva Granada, Bogotá (Colombia).

5. Docente de la Universidad Militar Nueva Granada, Bogotá (Colombia). tes fueron dismenorrea $(63,9 \%)$, seguido de dolor pélvico (35,2\%). Las tecnologías diagnósticas más utilizadas fueron el ultrasonido pélvico en un 92,1\% y la resonancia magnética nuclear en un 74,6\% de los casos. La histeroscopia y laparoscopia son poco utilizados en el diagnóstico.

Conclusión: el SHWW es una entidad poco frecuente, debe hacer parte del estudio complementario de la agenesia renal del paciente pediátrico y del diagnóstico diferencial de la dismenorrea primaria en pacientes en la adolescencia. Se requiere evaluar con estudios de cohorte más grandes la utilidad de la histeroscopia en estas pacientes.

Palabras clave: riñón único, vagina, anomalías urogenitales, conductos paramesonéfricos.

\section{ABSTRACT}

Objective: To report a case of Herlyn-WernerWunderlich syndrome (HWWS) and conduct a review of the literature to determine clinical and imaging findings in these patients.

Material and methods: A 16-year-old female patient who presented to a military hospital in Bogota complaining of recurrent pelvic pain, receiving a final diagnosis of HWWS. A systematic search was conducted in the different databases of systematic reviews, cohort studies, case series and case reports of patients of any age diagnosed with HWWS. Clinical presentation characteristics and the most frequent 
diagnostic technologies used were obtained. A narrative summary of the findings is presented.

Results: Overall, 77 publications with a total of 676 patients were included. The most frequent symptom was dysmenorrhea (63.9\%), followed by pelvic pain (35.2\%). The most frequently used diagnostic technologies were pelvic ultrasound in $92.1 \%$ of cases and nuclear magnetic resonance in $74.6 \%$. Hysteroscopy and laparoscopy are seldom used for diagnosing this condition.

Conclusion: HWWS is an infrequent disease condition. It must be part of the work-up for renal agenesis in pediatric patients and of the differential diagnosis in primary dysmenorrhea in adolescence. The role of hysteroscopy in this condition must be further assessed in larger cohort studies.

Keywords: Solitary kidney, vagina, urogenital abnormalities, mullerian ducts.

\section{INTRODUCCIÓN}

Las anormalidades müllerianas representan un amplio espectro de anomalías que incluyen agenesia o duplicación uterina y vaginal, y su incidencia se encuentra entre el 0,17 \% en la población general y 3,5 \% en mujeres infértiles (1). Entre las anomalías müllerianas se encuentra el síndrome de hemivagina obstruida y agenesia renal ipsilateral (OHVIRA por sus siglas en inglés), término que fue sugerido en 2007 para describir la asociación entre anomalías uterinas obstructivas y renales, como displasia o atrofia renal, riñón pélvico y uréter ectópico (2). Esta nueva denominación permite la inclusión de otras anomalías uterinas distintas al útero bicorpóreo, que se presentan en hasta el 22\% de los casos (3).

El síndrome de Herlyn Werner Wunderlich (SHWW) corresponde al $77 \%$ de los OHVIRA (4), consistente en la triada de útero bicorpóreo acompañado de hemivagina obstruida y agenesia renal ipsilateral. Este fue descrito por primera vez en 1922 (5); sin embargo, lleva su nombre por la publicación de Herlyn y Werner en 1971 (6), y de Wunderlich en 1976 (7). La frecuencia del síndrome de Herlyn-Werner-Wunderlich no es conocida, pero cada vez se está identificando más, aún en mujeres prepúberes, con las ayudas diagnósticas no invasivas (8).

No se conoce exactamente su etiología y patogenia, pero se atribuye al desarrollo anómalo de los conductos paramesonéfricos (de Müller) y de los conductos mesonéfricos (de Wolff). De estos últimos se forma la yema ureteral que induce la formación del riñón a partir del blastema metanéfrico, por lo cual la anomalía en los conductos mesonéfricos puede causar agenesia renal unilateral; además, participan en la fusión central de los conductos paramesonéfricos y el contacto de estos con el seno urogenital, de manera que su alteración puede desarrollar útero bicorpóreo y hemivagina obstruida (9).

La presentación habitual es durante la adolescencia con dolor pélvico progresivo, dismenorrea y ciclos irregulares, aunque pueden ser regulares si la obstrucción es incompleta (2). Por otra parte, el diagnóstico es imagenológico, la precisión de la resonancia magnética nuclear (RMN) en el diagnóstico de malformaciones uterinas puede llegar hasta el $100 \%$, puede adicionar información más precisa sobre la morfología uterina, continuidad del lumen vaginal y la naturaleza del contenido líquido, y con hallazgos de la triada: útero bicorpóreo, hemivagina obstruida y agenesia renal ipsilateral (10). El tratamiento es quirúrgico con resección de tabique vaginal y drenaje de hematocolpos (11), con lo cual se busca el alivio de los síntomas y garantizar resultados reproductivos exitosos (12).

Por ser una patología poco frecuente es importante que, tanto el pediatra como el ginecólogo, piensen en ella para una detección temprana en las niñas y en las adolescentes que presentan agenesia renal, ya que un retraso en el diagnóstico y en el tratamiento quirúrgico definitivo de la condición pueden llevar a un mal pronóstico en términos de fertilidad; por lo tanto, el objetivo de este artículo es presentar un reporte de caso de SHWW tipo 1.1 (según clasificación de Zhu L) (11) y hacer una revisión de la literatura sobre el diagnóstico inmunológico y quirúrgico utilizado en las pacientes con SHWW. 


\section{PRESENTACIÓN DEL CASO}

Mujer de 16 años quien es remitida de la región oriental de Colombia, en noviembre del 2020, por cuadro clínico de 8 meses de evolución que se exacerbó en los últimos 5 días, presentó dolor pélvico tipo cólico de predominio en epigastrio, de intensidad moderada a severa, asociado a leucorrea fétida, al servicio de ginecología del Hospital Militar Central (HMC), institución general de referencia ubicada en Bogotá que atiende población afiliada al régimen especial de salud de las fuerzas militares en el país. Como antecedente, la paciente había sido diagnosticada con agenesia renal izquierda, además presentaba dismenorrea desde la menarquia y planificaba con anticonceptivos inyectables. Había consultado a otra institución dos meses antes por sintomatología similar donde realizan diagnóstico de enfermedad pélvica inflamatoria estadio II, allí le realizaron laparotomía encontrando una pelvis sellada por múltiples adherencias; realizaron salpingectomía izquierda y liberación de adherencias, con mejoría parcial de los síntomas. Al examen físico de ingreso se encontró una paciente con $46 \mathrm{~kg}$ de peso y $150 \mathrm{~cm}$ de talla, se encontraba en aceptables condiciones generales, con taquicardia, y sin fiebre. En la valoración abdominal se evidenció leve dolor generalizado, sin signos de irritación peritoneal. En el examen ginecológico, en la especuloscopía evidencia abombamiento de la pared vaginal izquierda, con descarga vaginal amarilla, no fétida. En el tacto vaginal se encuentra induración en el tercio distal de la pared vaginal lado izquierdo, de aproximadamente $4 \mathrm{~cm}$, que se extiende hacia el fondo del saco posterior izquierdo. Cérvix posterior cerrado, no doloroso a la movilización. Se realizan paraclínicos encontrando hemograma con leucocitos $10.400 / \mathrm{mm} 3$ con neutrófilos $56 \%$, hemoglobina $11,1 \mathrm{mg} / \mathrm{dl}$ y plaquetas en $322.000 / \mathrm{mm} 3$, parcial de orina y gram de orina no sugestivos de infección, reactante de fase aguda negativo, prueba treponémica negativa, VIH no reactivo, gonadotropina coriónica cuantitativa negativo. En la ultrasonografía abdominal total se observó riñón único derecho, hipertrofia renal derecha compensadora. En la ultrasonografía transvaginal se encontró útero con presencia de dos cavidades hacia fondo uterino, separadas entre sí por tejido miometrial y unidas hacia la región ístmica, al

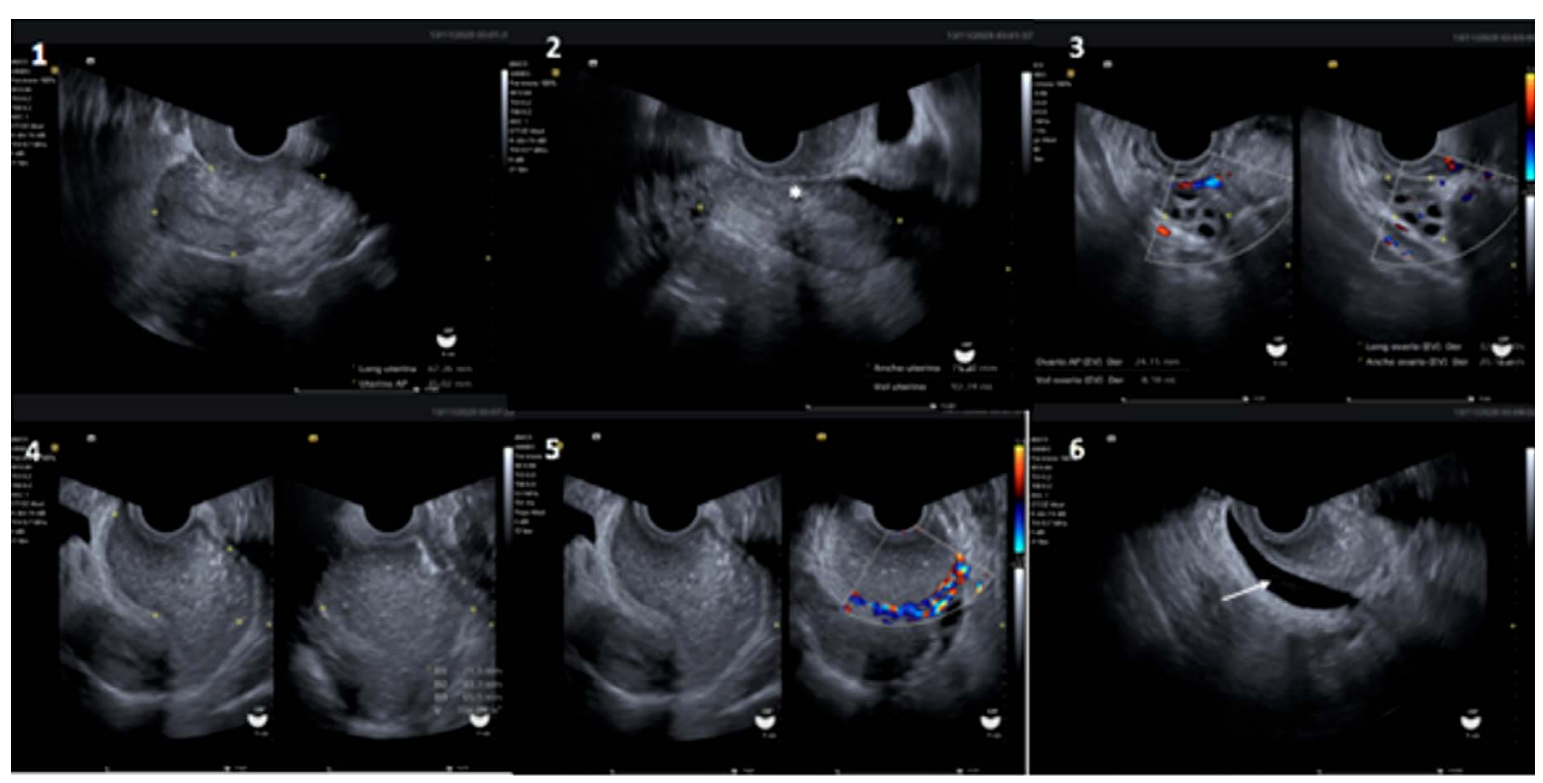

Figura 1. Imágenes de ecografías obtenidas en paciente con diagnóstico de SHWW mediante exploración transvaginal. 1. Hemiútero derecho con cérvix funcional. 2. Útero bicorpóreo con aparente unión entre hemiuteros en región ístmica.

3. Ovario derecho de aspecto ecográfico normal. 4. Colección hacia región cervicovaginal con contenido particulado 5. Valoración con doppler color. La colección presenta captación periférica fuerte. 6. La flecha señala imagen anecoica alargada, posible remanente ureteral.

Fuente: elaboración propia. 
parecer compartiendo un mismo cérvix, hacia cuello uterino, imagen ovalada con bordes bien definidos y contenido de ecogenicidad heterogénea, particulada que puede corresponder a colección en la región parauterina izquierda que mide $71,5 \times 65,5 \times 44,3$ mm para un volumen de $106 \mathrm{cc}$, que presentaba captación a la coloración Doppler en su periferia (score 3). Ovario derecho sin alteración y ovario izquierdo no visualizado (Figura 1).

Con diagnostico presuntivo de colección pélvica secundaria a enfermedad pélvica inflamatoria, se inició manejo antibiótico con clindamicina $460 \mathrm{mg}$ cada 6 horas y gentamicina 160 mg cada día, y se realizó laparotomía exploratoria el mismo día del ingreso, encontrando útero bicorpóreo U3, síndrome adherencial con adherencias firmes desde el colon sigmoide a la cara posterior de la vagina, vejiga ascendida y adherida a cara anterior de útero, no fue posible valorar ovario izquierdo por adherencias firmes de colon sigmoide a pared abdominal, sin líquido libre ni masas o colecciones dentro de la cavidad abdominal. Ante ausencia de diagnóstico definitivo, se lleva en el posoperatorio a resonancia magnética nuclear (RMN) que reporta útero bicorpóreo U3c, con cavidad vaginal tubular, y una colección lateral a la izquierda de la vagina (Figura 2).

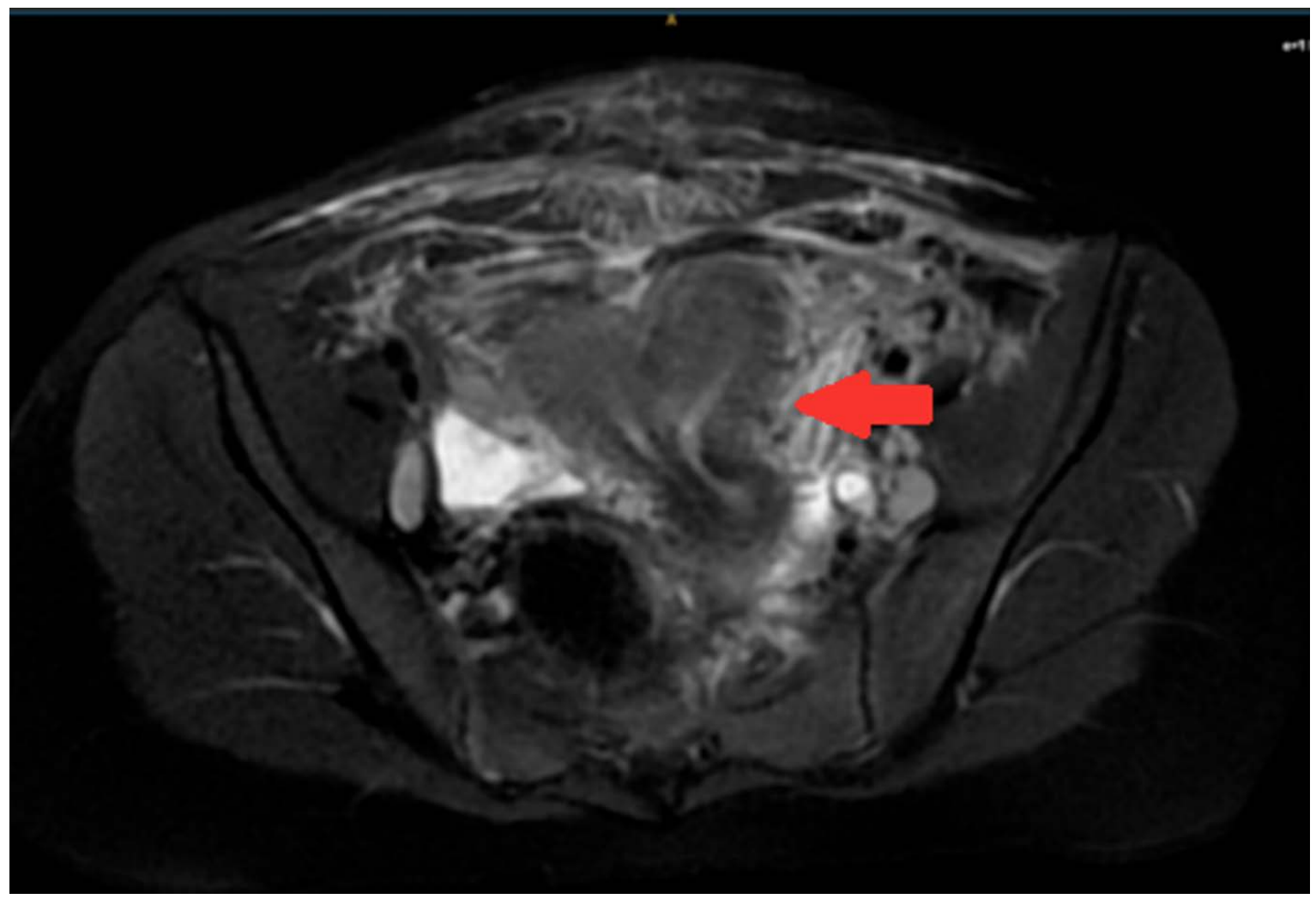

Figura 2. RMN imagen T1, se observa (flecha) dos cavidades uterinas completamente separadas, clasificado como útero bicorpóreo completo ESHRE/ESGE: U3b en paciente con SHWW.

Fuente: elaboración propia.

También se visualiza una segunda colección de contenido líquido, alargada, que se extiende hacia la hemipelvis izquierda y transcurre adyacente a los vasos ilíacos, con su extremo distal en el aspecto caudal y posterior de la vejiga (Figura 3 y 4). Se consideró que esta última imagen podría corresponder a remanente ureteral. Con los hallazgos anteriormente mencionados, y tomando en cuenta la RMN y el ultrasonido transvaginal, se considera que la colección pélvica podría corresponder a hemivagina izquierda obstruida, acompañada de agenesia renal ipsilateral y útero bicorpóreo U3, con diagnóstico de SHWW. 


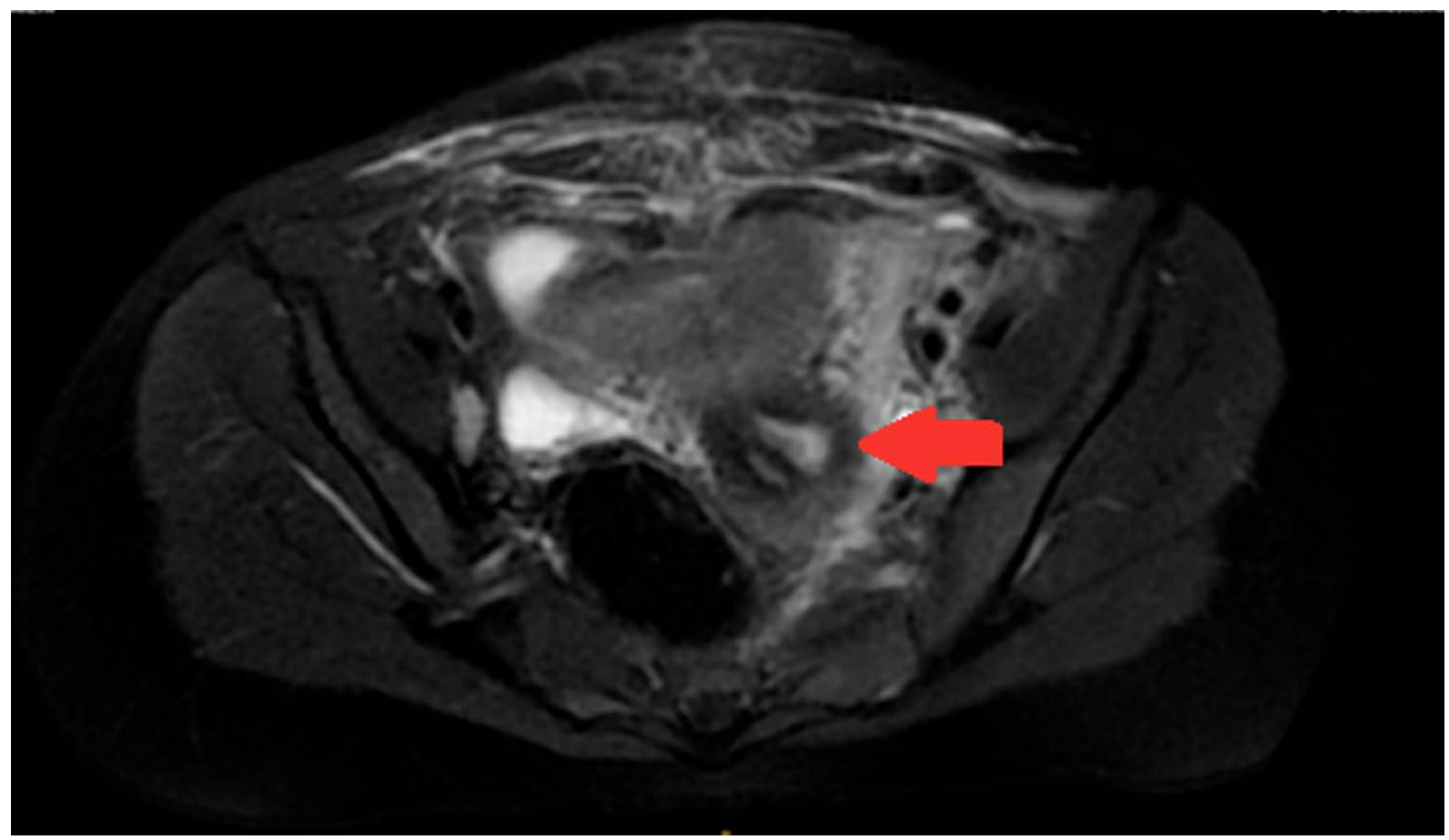

Figura 3. RMN imagen T, se observa (flecha) dos canales cervicales completamente separados por un septo, cérvix doble clasificación ESHRE/ESGE: C2 en paciente con SHWW.

Fuente: elaboración propia.

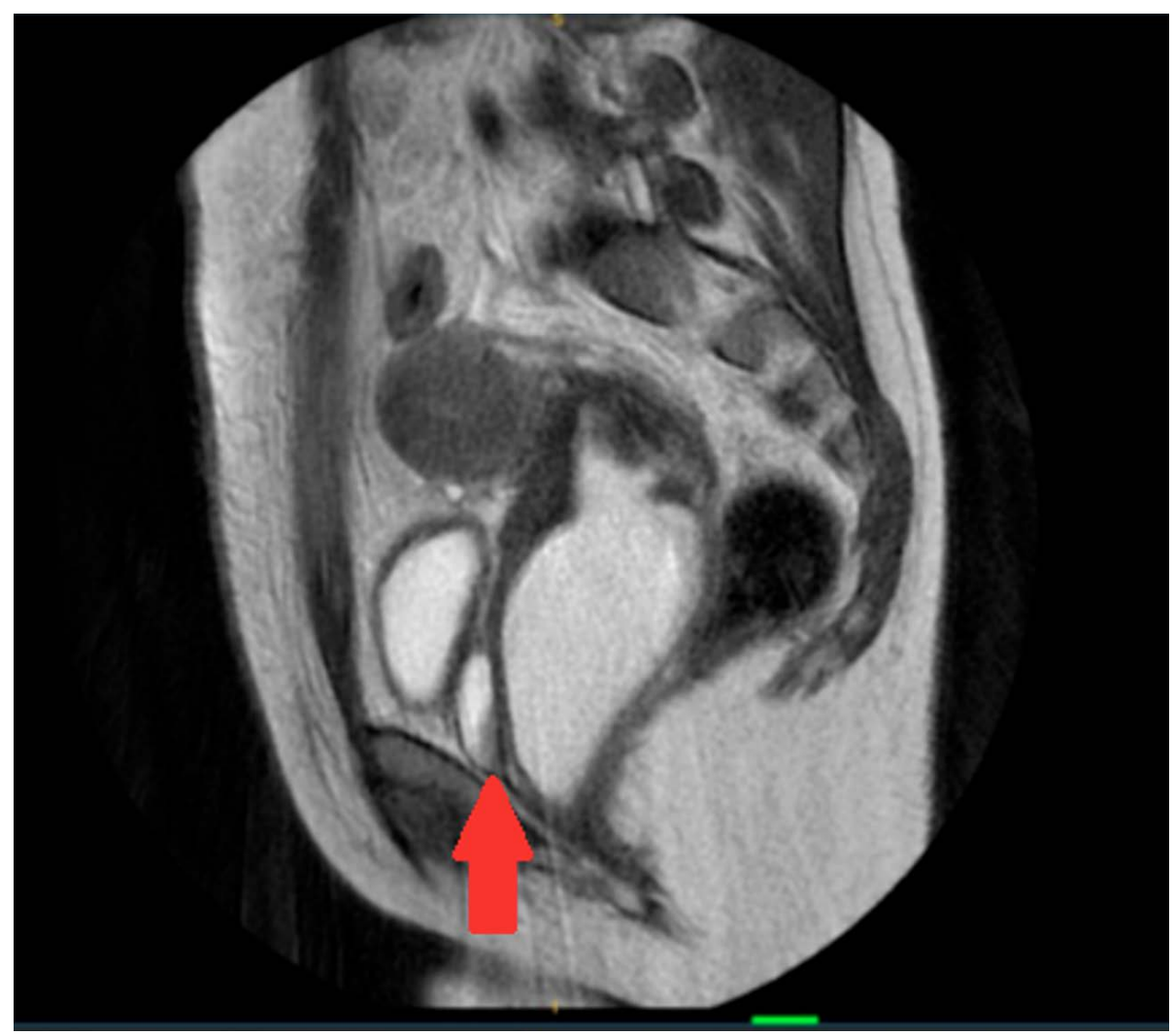

Figura 4. RMN imagen T2 corte sagital, se observa (flecha) septo longitudinal que separa hemivagina derecha con obstrucción, clasificación ESHRE/ESGE V2 en paciente con SHWW.

Fuente: elaboración propia. 
La paciente fue llevada a resección de tabique vaginal por vaginoscopia e histeroscopía diagnóstica al tercer día del ingreso. El procedimiento se realizó bajo anestesia general, se procedió con vaciamiento vesical con sonda nelaton, y posteriormente se hizo vaginoscopia e histeroscopía de hemiútero derecho con histeroscopio de Bettocchi; como hallazgos intraoperatorios se encontró cérvix derecho de aspecto sano, hemiútero derecho con cavidad endometrial vacía, endometrio sin alteraciones, ostium derecho visible; abombamiento de vagina en pared lateral izquierda y anterior obstruida por septo vaginal oblicuo. Ante la presencia de septo descrito se decide realizar apertura de este con resectoscopio monopolar con glicina como medio de distensión; se observó salida de material hemopurulento fétido, se realizó drenaje y lavado de vagina y se completó la resección del septo (Figura 5). Se realizó histeroscopía diagnóstica con histeroscopio de Bettochi de hemiútero izquierdo, se identificó cérvix y hemiútero izquierdo de aspecto hipotrófico, con cavidad endometrial vacía y pequeña. El ostium izquierdo no fue visualizado. Se dejó una sonda Foley número 14 con el balón inflado sobre 10 cc de SSN en hemivagina izquierda para disminuir el riesgo de nueva obstrucción. Se completó el manejo de antibiótico con clindamicina y gentamicina por 72 horas intrahospitalaria, la paciente presentó evolución clínica satisfactoria posterior al procedimiento, por lo que se da egreso al día siguiente.
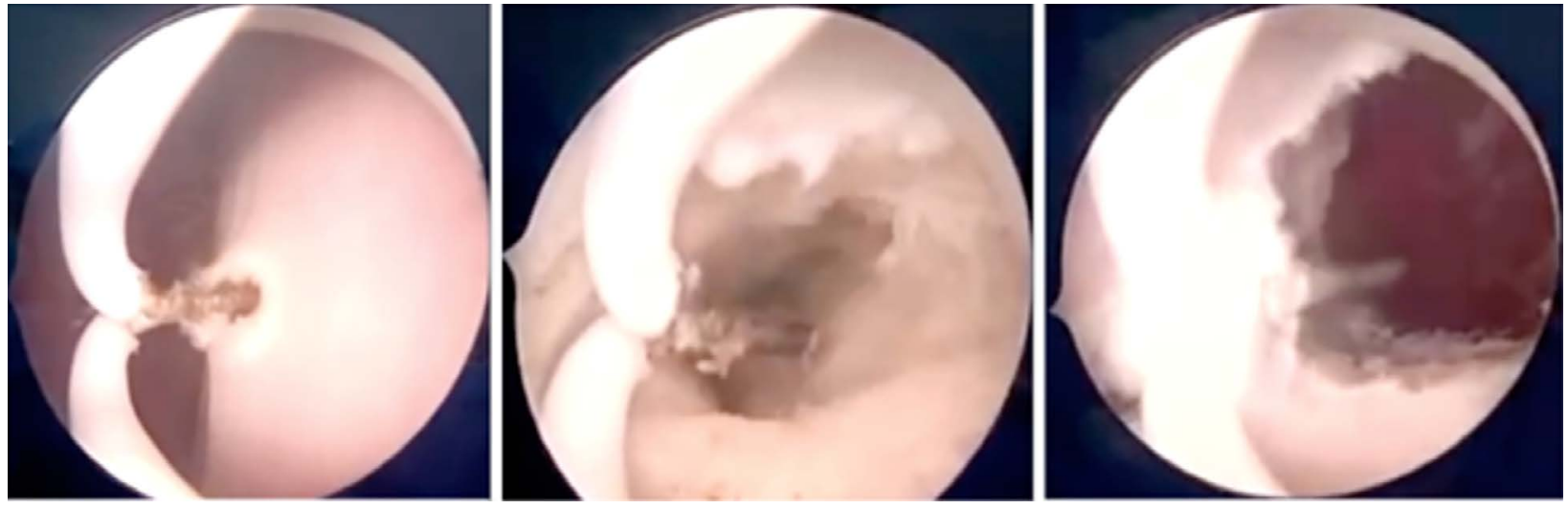

Figura 5. Visualización histeroscópica de apertura y resección del septo vaginal oblicuo en paciente con SHWW. Fuente: elaboración propia.

Se realizó control a los 15 días posteriores, donde se retiró la sonda Foley con evidencia de permeabilidad de vagina, con alivio completo de los síntomas y se dio inicio al método anticonceptivo tipo implante subdérmico; la paciente no realizó más controles en la institución por vivir fuera de la ciudad de Bogotá.

Aspectos éticos. Para el reporte de caso se contó con el consentimiento informado de la paciente y madre como representante legal. Se garantiza la confidencialidad de la información. Para la publicación del reporte de caso se cuenta con autorización de la paciente y su acudiente para el uso de datos de su historia clínica y material fotográfico y bajo la confidencialidad de la información obtenida, se aclaran dudas sobre el reporte de caso y su publicación.

\section{MATERIALES Y MÉTODOS}

Se realizó una búsqueda sistemática de la literatura en la base de datos MEDLINE, se utilizaron los términos MeSH "Agenesia renal”, "vagina", "malformación urogenital", "conductos mullerianos" y "útero". Se seleccionaron artículos originales sin restricción de lengua ni año de publicación. Como criterio de inclusión se abarcaron revisiones sistemáticas, ensayos clínicos, estudios observacionales, series de casos y reportes de casos, cuya población de estudio fueran pacientes con diagnóstico de SHWW en cualquier edad. Se buscó en cada uno de los estudios información concerniente al diseño, país, población incluida, edad media al diagnóstico, síntoma principal, tipo de tecnología diagnóstica, hallazgos imagenológicos, 
lateralidad y factores asociados. El único criterio de exclusión fue no tener acceso al texto completo. Se hace resumen narrativo de los hallazgos.

\section{RESULTADOS}

Se identificaron 91 títulos, de los cuales se descar- taron ocho; siete por no contar con pacientes con diagnóstico de SHWW y uno por corresponder a un video artículo. Posteriormente, nueve estudios fueron excluidos por no tener acceso al texto completo; finalmente, se incluyeron 74 publicaciones que cumplieron con los criterios de inclusión (Tabla 1).

\begin{tabular}{|c|c|c|c|c|c|c|c|}
\hline Referencia & Autor año & País & $\begin{array}{l}\text { Número } \\
\text { de casos }\end{array}$ & $\begin{array}{c}\text { Edad de } \\
\text { diagnóstico } \\
\text { (años) }\end{array}$ & Síntoma predominante & Prueba diagnóstica & Observaciones \\
\hline 4 & Fedele 2013 & Italia & 87 & $\begin{array}{l}\text { "Edad media 20,7 } \\
\text { rango }(11-42) "\end{array}$ & $\begin{array}{l}\text { Dismenorrea (94\%), manchado (41 \%), } \\
\text { dolor pélvico crónico (24\%), flujo vaginal (14 } \\
\%) \text {, dispareunia (14\%), fiebre (3\%) y dolor } \\
\text { abdominal agudo (2\%) }\end{array}$ & $\begin{array}{c}\text { Ecografía pélvica y resonancia } \\
\text { magnética, } 7 \% \text { histeroscopía } \\
\text { diagnóstica }\end{array}$ & $14 \%$ endometriosis \\
\hline 10 & Zhang 2019 & China & 19 & $\begin{array}{l}\text { "Edad media de } 17,6 \\
\text { rango }(11 \text { y } 28)\end{array}$ & $\begin{array}{l}68 \% \text { de pacientes presentado con amenorrea } \\
\text { primaria y dolor abdominal inferiores periódico, } \\
15 \% \text { pacientes con flujo vaginal anormal, } \\
\text { retención urinaria y } 15 \% \text { pacientes con masa } \\
\text { pélvica }\end{array}$ & $\begin{array}{l}\text { Ecografía pélvica y resonancia } \\
\text { magnética }\end{array}$ & \\
\hline 11 & Zhu L 2015 & China & 78 & $\begin{array}{l}\text { Tipo } 1 \text { edad media } \\
21,3 \text { Tipo } 2 \text { edad } \\
\text { media } 25,74\end{array}$ & $\begin{array}{c}1 \text { dismenorrea } 79 \%, 2 \text { descarga mucopurulenta } \\
51 \% \text { como síntomas principales }\end{array}$ & & $\begin{array}{l}52 \text { embarazos, ipsilateral } 19 \\
(37 \%) \text { u contralateral } 33 \\
(64 \%), 14 \% \text { exploracion } \\
\text { abdominal por laparotomia o } \\
\text { laparoscopia }\end{array}$ \\
\hline 12 & Cándenas 2019 & España & 1 & 37 & Asintomática & Ecografía pélvica y RMN & $\begin{array}{l}\text { Embarazo complicado con } \\
\text { RPMO }\end{array}$ \\
\hline 13 & Zhang 2020 & China & 40 & $\begin{array}{l}\text { Edad media } 20 \text { rango } \\
(11-53)\end{array}$ & $\begin{array}{l}50 \% \text { dolor pélvico y dismenorrea, } 35 \% \text { HUA: } \\
\text { irregular, } 5 \% \text { disuria, } 5 \% \text { dispareunia y } 20 \% \\
\text { asintomáticas (incidental) }\end{array}$ & $\begin{array}{l}\text { RMN todas y ecografía } \\
\text { transabdominal abdominal } 25 \mathrm{y} \\
\text { ecografía transvaginal } 15\end{array}$ & \\
\hline 14 & Wang 2014 & Alemania & 61 & $\begin{array}{l}\text { Edad media Tipo I; } \\
\text { 14,2 Tipo II } 20.9 \\
\text { Tipo III } 19,5\end{array}$ & $\begin{array}{c}\text { Dismenorrea tipo I } 92 \% \text { tipo II } 57,1 \% \text {, tipo } \\
\text { III } 50 \% \text {, masa quistica en pared vaginal tipo I } \\
60 \% \text {, tipo II } 17.9 \% \text {, tipo II } 37,5 \% \text {, descarga } \\
\text { vaginal anormal Tipo I } 24 \% \text {, tipo II } 75 \% \text {, tipo } \\
\text { III } 50 \% \text {. }\end{array}$ & Ecografía pélvica & $\begin{array}{l}11 \text { laparotomias, } 5 \text { abscesos } \\
\text { pélvicos, } 5 \text { estenosis y } \\
\text { reintervención }\end{array}$ \\
\hline 15 & Tong 2013 & China & 70 & $\begin{array}{c}\text { Edad media } 21,3 \\
\text { rango (10-50 años) }\end{array}$ & Disemnorrea $90 \%$ & $\begin{array}{l}\text { Ecografía pélvica y } 52 \% \\
\text { confirmación con RMN }\end{array}$ & $\begin{array}{l}12,9 \% \text { de laparotomias por } \\
\text { absceso, endometrioma o } \\
\text { adherencias } 14,20 \% \text { historia } \\
\text { de EPI, } 17,1 \% \text { endoemtrio- } \\
\text { sis, } 58 \text { embarazos, ipsilateral } \\
\text { en } 36,5 \% \text {, contralateral } \\
63,5 \%\end{array}$ \\
\hline 16 & Yavuz 2015 & Turquía & 13 & $\begin{array}{c}\text { Edad media } 12,9 \\
\text { rango }(12-23)\end{array}$ & Todas dolor abdomen inferior o dolor pélvico & $\begin{array}{c}\text { Ecografía pélvica y } 77 \% \text { RMN } \\
\text { adicional }\end{array}$ & 2 partos normales, 2 abortos \\
\hline 17 & Mohd 2018 & India & 4 & $\begin{array}{lll}\text { caso } & 1 & 18 \\
\text { caso } & 2 & 14 \\
\text { caso } & 3 & 26 \\
\text { caso } & 4 & 13\end{array}$ & Dolor pélvico y dismenorrea & Ecografía pélvica y RMN & \\
\hline 18 & Gungor 2020 & Turquía & 32 & $\begin{array}{l}\text { Edad media } 16,8 \\
\text { rango }(14-32)\end{array}$ & Dolor pélvico y dismenorrea & Ecografía pélvica y RMN & \\
\hline 19 & Kapczuk 2017 & Polonia & 22 & $\begin{array}{l}\text { Edad media } 13,1 \\
\text { rango }(11,4 \text { y } 18,2)\end{array}$ & $\begin{array}{c}\text { Dolor pélvico y dismenorrea, } 4 \% \text { de retención } \\
\text { urinaria. }\end{array}$ & No reportan & \\
\hline 20 & Fascilla 2020 & Italia & 5 & $\begin{array}{ll}\text { caso } 1 & 26 \\
\text { caso } 2 & 21 \\
\text { caso } 3 & 12 \\
\text { caso } 4 & 16 \\
\text { caso } 5 & 23\end{array}$ & $\begin{array}{l}\text { Casos } 1 \text { y } 2 \text {. Dismenorrea, disparunia más } \\
\text { apalpable, caso } 3 \text { abdomen agudo, masa } \\
\text { palpable, caso } 4 \text { y } 5 \text { dismenorrea }\end{array}$ & $\begin{array}{l}\text { Ecografía pélvica transvaginal y } \\
\text { transrectal, RMN }\end{array}$ & $\begin{array}{l}1 \text { embarazo sin } \\
\text { complicaciones }\end{array}$ \\
\hline
\end{tabular}




\begin{tabular}{|c|c|c|c|c|c|c|c|}
\hline Referencia & Autor año & País & $\begin{array}{l}\text { Número } \\
\text { de casos }\end{array}$ & $\begin{array}{c}\text { Edad de } \\
\text { diagnóstico } \\
\text { (años) }\end{array}$ & Síntoma predominante & Prueba diagnóstica & Observaciones \\
\hline 21 & Gholoum 2006 & Canadá & 12 & $\begin{array}{l}\text { Edad media } 13 \text { rango } \\
(11-14)\end{array}$ & $\begin{array}{c}\text { Todas dolor abdominal y masa palpable } \\
\text { abdominal }\end{array}$ & $\begin{array}{c}11 \text { Ecografía pélvica } 2 \text { TAC y } 3 \\
\text { RMN }\end{array}$ & 2 abscesos intraabdominales \\
\hline 22 & Mallmann 2018 & Alemania & 1 & Neonatal & Asintomática & Ecografía pélvica & $\begin{array}{l}\text { Serie de } 20 \text { casos, solo un } \\
\text { paciente con SHHW }\end{array}$ \\
\hline 23 & Oestreich 2008 & Italia & 11 & $\begin{array}{l}\text { Edad media } 12,9 \\
\text { rango }(12-15)\end{array}$ & $\begin{array}{l}36 \% \text { HUA, } 36 \% \text { dismenorrea, } 27 \% \text { dolor } \\
\text { abdominal agudo }\end{array}$ & Ecografía pélvica & \\
\hline 24 & Liu 2021 & China & 37 & $\begin{array}{c}\text { Edad media } 23 \text { rango } \\
(16-26)\end{array}$ & Dismenorrea, dolor pélvico progresivo & Ecografía pélvica & \\
\hline 25 & Tong 2014 & China & 94 & $\begin{array}{l}\text { Edad media } 20,5 \\
\text { rango }(13-37)\end{array}$ & No reporta & No reportan & $19,1 \%$ endometriosis \\
\hline 26 & Tuna 2018 & Portugal & 1 & Neonatal & Hematolcopos & Ecografía pélvica y RMN & \\
\hline 27 & Nishu 2019 & Bangladesh & 1 & 15 & $\begin{array}{c}\text { Dismenorrea, dolor pélvico y fosa iliaca derecha } \\
\text { y hematocolpos }\end{array}$ & Ecografía pélvica y RMN & \\
\hline 28 & Fachin 2019 & Brasil & 1 & 12 & $\begin{array}{l}\text { Dismenorrea, dolor pélvico y sangrado } \\
\text { intermestrual }\end{array}$ & Ecografía pélvica, TAC y RMN & \\
\hline 29 & Mittal 2015 & India & 1 & 16 & Dismenorrea y dolor pélvico & Ecografía pélvica y RMN & \\
\hline 30 & Salastekar 2019 & EEUU & 1 & 16 & $\begin{array}{l}\text { Dispareunia, sangrado postcoital y } \\
\text { ocasionalmente con hematoquecia. }\end{array}$ & Ecografía pélvica y RMN & \\
\hline 31 & Jung 2017 & Korea & 1 & 22 & $\begin{array}{l}\text { Secreción vaginal fétida con sangrado } \\
\text { intermenstrual }\end{array}$ & Ecografía pélvica y RMN & piocolpos \\
\hline 32 & Gupta 2018 & EEUU & 1 & 16 & Dolor abdominal & Ecografía pélvica y RMN & Abdomen agudo \\
\hline 33 & Jia 2018 & China & 1 & 22 & Dolor pélvico & Ecografía pélvica & $\begin{array}{l}\text { embarazo con aborto } \\
\text { espontaneo }\end{array}$ \\
\hline 34 & Cappello 2018 & Italia & 1 & 28 & $\begin{array}{l}\text { "Dolor pélvico agudo severo } \\
\text { menarquia, debido a dolor pélvico agudo severo" }\end{array}$ & $\begin{array}{l}\text { RMN y se confrimó por } \\
\text { laparoscopia }\end{array}$ & Con ectrodactilia. \\
\hline 35 & Reis 2016 & Portugal & 1 & 27 & Dispareunia & Ecografía pélvica y RMN & Embarazo sin complicaciones \\
\hline 36 & Miyazaki 2019 & Japón & 1 & 20 & Dolor pélvico & Ecografia pélvica y RMN & 3 perdidas espontaneas \\
\hline 37 & Angotti 2015 & Italia & 1 & 3 & Masa abdominal, dolor pélvico & Ecografía pélvica y RMN & \\
\hline 38 & Vázquez 2020 & España & 1 & Neonatal & Asintomática & Ecografía pélvica & \\
\hline 39 & Rastogi 2010 & India & 1 & 14 & Dismenorrea & Ecografía pélvica y RMN & Abdomen agudo \\
\hline 40 & $\begin{array}{c}\text { Tangshewinsirikul } \\
2020\end{array}$ & Tailandia & 1 & 33 & Dolor pélvico, leucorrea & Ecografía pélvica y RMN & Embarazo y piocolpus \\
\hline 41 & Gungor 2014 & Turquía & 1 & 13 & $\begin{array}{l}\text { Dolor abdominal agudo y masa pélvica } \\
\text { una masa pélvica palpable }\end{array}$ & Ecografía pélvica y RMN & Abdomen agudo \\
\hline 42 & Yakıştıran 2016 & Turquía & 1 & 13 & Dismenorrea & Ecografía pélvica y RMN & \\
\hline 43 & Takagi 2010 & Japón & 2 & $\begin{array}{l}\text { "caso } 1-14 \\
\text { caso } 2-52 "\end{array}$ & $\begin{array}{l}\text { "caso } 1 \text { Dolor abdominal y dismenorrea } \\
\text { caso } 2 \text { Dolor abdominal y leucorrea" }\end{array}$ & RMN & \\
\hline 44 & Sen 2013 & India & 1 & 13 & Dolor pélvico severo, dismenorrea & Ecografía pélvica y RMN & \\
\hline 45 & Mandava 2012 & India & 1 & 14 & Retención urinaria, masa pélvica & $\begin{array}{l}\text { Ecografía pélvica, } \mathrm{RMN} \text { y } \\
\text { confirmación por laparoscopia }\end{array}$ & \\
\hline 46 & Bajaj 2012 & India & 1 & 14 & Dolor pélvico & Ecografía pélvica y RMN & \\
\hline
\end{tabular}

Continuación Tabla 1 


\begin{tabular}{|c|c|c|c|c|c|c|c|}
\hline Referencia & Autor año & País & $\begin{array}{l}\text { Número } \\
\text { de casos }\end{array}$ & $\begin{array}{c}\text { Edad de } \\
\text { diagnóstico } \\
\text { (años) }\end{array}$ & Síntoma predominante & Prueba diagnóstica & Observaciones \\
\hline 47 & Jindal 2009 & India & 1 & 14 & Dismenorrea & $\begin{array}{l}\text { Ecografía pélvica y laparoscopia } \\
\text { diagnóstica }\end{array}$ & \\
\hline 48 & Vivier 2011 & Francia & 1 & 5 & Diagnóstico prenatal y confirmación a los 5 años & Ecografía pélvica y RMN & \\
\hline 49 & Mishra 2014 & Australia & 2 & $\begin{array}{l}\text { "caso } 1-13 \\
\text { caso } 2-13 "\end{array}$ & Dismenorrea & Ecografía pélvica y RMN & \\
\hline 50 & Wu 2012 & Taiwan & 1 & Neonatal & Masa pélvica & Ecografía pélvica & \\
\hline 51 & Cozzolino 2014 & Italia & 1 & 12 & Dismenorrea & $\mathrm{RMN}$ & $\begin{array}{l}\text { Dos embarazos, RPMO, } \\
\text { parto pretérmino }\end{array}$ \\
\hline 52 & Sanghvi 2011 & India & 1 & 4 & Dolor abdominal, disuria & Ecografía pélvica y TAC & \\
\hline 53 & Pereira 2014 & EEUU & 1 & 25 & Dismenorrea & Ecografía pélvica y RMN & \\
\hline 54 & Han 2013 & Corea & 1 & $\begin{array}{c}\text { Diagnóstico antenatal } \\
20.1 \mathrm{ss}\end{array}$ & Diagnóstico prenatal & Ecografía osbtetrica antenatal & \\
\hline 55 & $\begin{array}{l}\text { Wozniakowska } \\
2014\end{array}$ & Polonia & 1 & 14 & Leucorrea & Ecografía transrectal y RMN & \\
\hline 56 & Dorais 2011 & EEUU & 1 & 14 & Dolor abdominal & Ecografía pélvica & quiste de Gartner \\
\hline 57 & Watanabe 2012 & Japón & 2 & $\begin{array}{l}\text { Caso } 133 \\
\text { Caso } 253\end{array}$ & No reporta & RMN & $\begin{array}{c}\text { Caso } 1 \text { adenocarcinoma } \\
\text { endoemtrioide Caso } 2 \text { carci- } \\
\text { noma de celuals claras }\end{array}$ \\
\hline 58 & Liang 2020 & Taiwan & 1 & 19 & Dolor abdominal, dismenorrea & Ecografía pélvica & \\
\hline 59 & Nabeshima 2013 & Japón & 1 & 12 & Dolor abdominal y dismenorrea & $\mathrm{RMN}$ & \\
\hline 60 & Asha 2008 & India & 1 & 17 & Dolor abdominal y dismenorrea & Ecografía pélvica y laparoscopia & \\
\hline 61 & Roth 2009 & Canadá & 1 & 3 & Dolor abdominal y masa pelvica & Ecografía pélvica & 4 pero solo 1 SHWW (267) \\
\hline 62 & Rana 2008 & India & 1 & 23 & Dolor abdominal & Ecografía pélvica y RMN & Embarazo \\
\hline 63 & Kumar 2015 & India & 1 & 14 & Retencion urinaria aguda, dismenorrea & Ecografía pélvica y RMN & \\
\hline 64 & Güdücü 2012 & Turquía & 2 & 1 caso 192 caso 21 & $\begin{array}{l}\text { 1ra dismenorrea, dispareunia 2da leucorrea } \\
\text { fetida }\end{array}$ & Ecografía pélvica y RMN & \\
\hline 65 & Sarac 2009 & Turquía & 1 & 25 & dismenorrea & RMN & \\
\hline 66 & Noviello 2018 & Italia & 6 & $\begin{array}{c}\text { Edad media } 9 \text { años } \\
\text { Rango ( } 2 \text { meses a } \\
15 \text { años })\end{array}$ & $40 \%$ masa pélvica y $60 \%$ dolor abdominal & RMN & \\
\hline 67 & Yung 2016 & China & 1 & 42 & HUA ciclos irregulares y anemia & Ecografía pélvica y RMN & \\
\hline 68 & Aveiro 2011 & Portugal & 1 & 13 & Dolor pélvico, dismenorrea & Ecografía pélvica, TAC y RMN & \\
\hline 69 & Beer 2013 & EEUU & 1 & 13 & Dolor vaginal, masa vaginal palpable & TAC y RMN & \\
\hline 70 & Schutt 2012 & EEUU & 3 & $\begin{array}{c}1 \text { caso } 15,2 \text { caso } 14 \\
3 \text { caso } 11\end{array}$ & Dolor pélvico & $\begin{array}{c}1 \text { Ecografía pélvica y RMN, } 2 \\
\text { Ecografía pélvica, } 3 \text { Ecografía } \\
\text { pelvica, RMN y laparoscopia } \\
\text { diagnóstica }\end{array}$ & \\
\hline 71 & Del Vescovo 2012 & Italia & 2 & 1 caso 16,2 caso 15 & Dismenorrea & $\begin{array}{c}\text { Caso 1: Ecografía pélvica y RMN, } \\
\text { Caso } 2 \text { RMN }\end{array}$ & \\
\hline 72 & Cox 2012 & EEUU & 1 & 17 & dolor pelvico, masa palpable & Ecografia pelvica y RMN & piocolpos \\
\hline
\end{tabular}

Continuación Tabla 1 


\begin{tabular}{|c|c|c|c|c|c|c|c|}
\hline 73 & Dogan 2016 & Turquía & 2 & $\begin{array}{l}\text { caso 1: } 11 \text { caso 2: } \\
11 \text { años }\end{array}$ & Dolor pélvico y dismenorrea & RMN & \\
\hline 74 & Karaca 2015 & Turquía & 1 & 13 & Dolor abdominal & Ecografía pélvica y RMN & \\
\hline 75 & Moshiri 2012 & EEUU & 1 & 31 & Asintomática & $\mathrm{RMN}$ & Infertilidad primaria \\
\hline 76 & Khaladkar 2016 & India & 1 & 13 & Dolor pélvico & $\begin{array}{c}\text { Histeroscopia, ecografia pélvica } \\
\text { y RMN }\end{array}$ & \\
\hline 77 & $\begin{array}{l}\text { Kozlowski } \\
2020\end{array}$ & Polonia & 2 & $\begin{array}{c}1 \text { caso } 172 \\
\text { caso } 13\end{array}$ & Dolor pélvico y dismenorrea & Ecografía pélvica y RMN & piocolpos \\
\hline 78 & $\begin{array}{l}\text { Ellspermann } \\
\quad 2017\end{array}$ & EEUU & 1 & 12 & Dolor pélvico & Ecografía pélvica & \\
\hline 79 & Hamidi 2018 & Afganistan & 1 & 19 & Dolor pélvico crónico y masa palpable & Ecografía pélvica y RMN & \\
\hline 80 & Meneses 2017 & Brasil & 1 & 12 & Dolor abdominal & Ecografía pélvica y RMN & $\begin{array}{l}\text { Absceso en remantente } \\
\text { mesonéfrico }\end{array}$ \\
\hline 81 & $\begin{array}{l}\text { Widyakusuma } \\
2018\end{array}$ & Indonesia & 1 & 23 & Dismenorrea & Ecografía pélvica, TAC y RMN & \\
\hline 82 & Tan 2020 & Singapur & 6 & $\begin{array}{c}4 \text { casos diagnóstico } \\
\text { perinatal } 2 \text { casos } 3 \\
\text { y } 12 \text { años }\end{array}$ & No reporta & Ecografía pélvica, un caso RMN & $\begin{array}{l}2 \text { excluidos por riñón } \\
\text { multiquístico }\end{array}$ \\
\hline
\end{tabular}

En las 74 publicaciones incluidas se encontraron 6 estudios observacionales retrospectivos (4, 13-17), 10 series de casos (10-11, 18-25) y 58 reportes de caso (12, 26-82), que incluyeron un total de 663 pacientes. La mayoría de las publicaciones fueron en India con 11 estudios (17,29,39,44-47,52,60,62,63), seguido de USA con 9 estudios $(30,32,53,56,69,70,72,75,78)$, China $(10,11,13,15,24,25,33,67)$ e Italia $(4,20,23,34,37,51,66,71)$ con 8 estudios cada uno. La serie de casos con mayor población fue publicada en China (25).

Tipo de población. La población incluida presenta una edad media al diagnóstico de 16,8 años (0 a 53 años). Un reporte de caso de diagnóstico ecográfico antenatal (54), 4 de diagnóstico neonatal $(22,26,38,50), 12$ entre 1 y 12 años $(28,37,48,51$ ,52,59,61,66,73,78,80,82), 26 entre 13 y 19 años $(27,29,30,32,39,41,42,44-47,49,55,56,58,60,63,68$ $72,74,76,77,79)$ y 15 reportes de mayores de 20 años (31,33-36,40,43,53,57,62,64,65,67,75,81).

Diagnóstico clínico. La aproximación diagnóstica varía según la edad de la paciente. En las pacientes prepúberes el principal hallazgo fue masa pélvica palpable compatible con hidrocolpos, acompañado del hallazgo de agenesia renal ipsilateral $(29,37,48,50,52,66)$. En algunos casos, el hallazgo de agenesia renal fue incidental en la etapa prenatal $(38,50,54)$, otros síntomas que reportaron fue la disuria $(37,52)$ y masa prolapsada por vagina (50). Sin embargo, a ninguna paciente se le realizó tamizaje de rutina por el hallazgo prenatal conocido.

Los síntomas más frecuentes entre las pacientes adolescentes son dismenorrea y dolor pélvico $(21,27-$ $31,32,51,55,56,64,67,68,69,71,72,74,75,78,79)$, solo un estudio reportó asociación a retención urinaria (19). En un estudio se realizó el diagnóstico durante el embarazo (62) asociado a sangrado vaginal. No se encontraron reportes de diagnóstico en pacientes postmenopáusicas.

A continuación, se exponen las características clínicas presentadas por las pacientes adultas con SHWW. El síntoma más frecuente es dismenorrea, con en el $64 \%$ de los casos (1215,17-20,24,28,29,36,39,41-43,49,51,53,60,63-65), seguida de dolor pélvico con el 35,2\% de los casos $(9,11,13,16,20,27,28,32,34,40,44,46,47,52,58,60$ 62,66,70-74,76-80). Se identificó endometriosis en un $10,3 \%$ de casos $(14,20,24,26,63)$. Otras manifestaciones reportadas en la literatura son descarga vaginal fétida en un estudio (16), y piocolpos en otro (56), y tres estudios informan pacientes asintomáticas $(10,13,75)$. Un total de 5 estudios informan como síntoma principal hemorragia uterina anormal $(12,23,31,32,67)$, pero esta fue principalmente manifestada en pacientes con septo parcial. Respecto a los signos clínicos 12 estudios presentan masa palpable en abdomen o pelvis $(14,20,21,32,37,41,45,50,58,61,66,72)$. 
El diagnóstico imagenológico fue realizado mediante ecografía pélvica transabdominal o transvaginal en el 92,1\% de los casos (12,14-18,20,21,24,26-32,3542,44-46,48-50,53,56,58,61-64,67,68,70-72,74,7782), complementado con resonancia magnética nuclear $(\mathrm{RMN})$ en el 74,6 \% (10,14-18,20,21,26$32,35-37,39,44-46,48,49,51,53,57,59,62-68,70$ 75,77,79-82); tomografía axial computarizada en el 1,7 \%, histeroscopía en el 1,7 \%, e histerosalpingografía en el 0,9\%. Solamente el 7,3\% de los casos fueron evaluados con un solo tipo de imagen, 3,8 \% con ecografía pélvica transabdominal o transvaginal $(24,38,50,56,58,61,78)$ y el $3,5 \%$ únicamente con RMN $(43,51,57,59,65,66,73,75)$.

Entre las características ecográficas reportadas se incluyen anomalías uterinas (útero bicorpóreo) y agenesia renal ipsilateral. Otros hallazgos fueron la presencia de un área anecoica o masa quística debajo del cérvix hasta la superficie perineal con regiones hiperecoicas intensas en forma de puntos, sugestivas de hematocolpos $(12,53,61)$; además, en algunos casos se reporta la presencia de hidrosalpinx (71).

Entre las características de la RMN para la evaluación de anormalidades müllerianas se observaron útero bicorpóreo y cuellos cervicales completamente separados, hendidura profunda externa del fondo hasta el nivel del cuello uterino (12,14-17,20,26-32,3537,39,44-46,51,53,57,59,62-68,70-75,77,79-82).

Histeroscopia y laparoscopia. Solo 3 publicaciones incluyen la laparoscopia en el diagnóstico del SHWW $(34,47,60)$ y un estudio previo con diagnóstico por histeroscopia (76), entre los hallazgos intraoperatorios un estudio reporta el drenaje de pus o sangre durante la punción de la masa paravaginal (10).

La lateralidad de la hemivagina obstruida y agenesia renal fue de predominio derecho, con un $59,6 \%$ de casos (n:241) (21,26,27,29,31-33,35,37$41,44,46,47,51-53,55,62,65,67-69,73-75,78-81)$ mientras que la izquierda presentó un 40,4 \% (n:164) $(20,28,30,34,42,45,48,54,58-61,63,70,72,76)$. Algunos estudios informan otras malformaciones asociadas, entre ellas ectrodactilia (34), túbulos colectores duplicados (9), persistencia de ducto mesonéfrico
(81), quiste de Gartner (56) y remanentes mesonéfricos (80).

\section{CONCLUSIONES}

El SHWW es una entidad poco frecuente que debe hacer parte del estudio complementario de la agenesia renal del paciente pediátrico y del diagnóstico diferencial de la dismenorrea primaria en pacientes en la adolescencia. Se requiere evaluar con estudios de cohorte más grandes la utilidad de la hieroscopia y la laparoscopía en estas pacientes.

\section{REFERENCIAS}

1. Nahum GG. Uterine anomalies. How common are they, and what is their distribution among subtypes? J Reprod Med. 1998;43(10):877-87

2. Siu A, Vargas V, Murcia FJ, Escassi A, Garrido JI, Antón M, et al. Síndrome de OHVIRA: características clínicas y complicaciones, nuestra experiencia. Cir Pediatr. 2019;32(1):11-16.

3. Haddad B, Barranger E, Paniel BJ. Blind hemivagina: long-term follow-up and reproductive performance in 42 cases. Hum Reprod. 1999;14(8):1962-4. doi: https://doi.org/10.1093/humrep/14.8.1962

4. Fedele L, Motta F, Frontino G, Restelli E, Bianchi S. Double uterus with obstructed hemivagina and ipsilateral renal agenesis: pelvic anatomic variants in 87 cases. Hum Reprod. 2013;28(6):1580-3. https://doi. org/10.1093/humrep/det081

5. Purslow C. A case of unilateral hematocolpos, hematometra and hematosalpinx. J Obstet Gynaecol Br Emp 1922;29(1): 643. https://doi. org/10.1111/j.1471-0528.1922.tb16100.x

6. Herlyn U, Werner H. Simultaneous occurrence of an open Gartner-duct cyst, a homolateral aplasia of the kidney and a double uterus as a typical syndrome of abnormalities. Geburtshilfe Frauenheilkunde 1971; 31(1):340-347

7. Wunderlich M. Unusual form of genital malformation with aplasia of the right kidney. Zentralbl Gynakol 1976; 988(1):559-562

8. Dietrich JE, Millar DM, Quint EH. Obstructive reproductive tract anomalies. J Pediatr Adolesc Gynecol. 2014;27(6):396-402. https://doi.org/10.1016/j. jpag.2014.09.001 
9. Acién P. Embryological observations on the female genital tract. Hum Reprod. 1992;7(4):437-45. https:// doi.org/10.1093/oxfordjournals.humrep.a137666

10. Zhang J, Xu S, Yang L, Songhong Y. MRI image features and differential diagnoses of Herlyn-Werner-Wunderlich syndrome, Gynecological Endocrinology.2019;40(9):1893-1900. https://doi.org/10.1 080/09513590.2019.1680623

11. Zhu L, Chen N, Tong J-L, Wang W, Zhang L, Lang J-H. New Classification of Herlyn-Werner-Wunderlich Syndrome. Chinese Medical Journal. 2015; 128 (2): 222225. https://doi.org/10.4103/0366-6999.149208

12. Cándenas LB, Davó DA, Peña M, Orlando JM, Díaz ACL. Diagnosis and gestational follow-up in a patient with Herlyn-Werner-Wünderlich syndrome, a case report. Taiwanese Journal of Obstetrics and Gynecology. 2019; 58(4): 560-565. https://doi.org/10.1016/j. tjog.2019.05.023

13. Zhang H, Ning G, Fu C, Bao L, Guo Y. Herlyn-WernerWunderlich syndrome: diverse presentations and diagnosis on MRI. Clin Radiol. 2020;75(6):480-487. https://doi.org/10.1016/j.crad.2020.01.016

14. Wang J, Zhu L, Lang J, Liu Z, Sun D, Leng J, et al. Clinical characteristics and treatment of HerlynWerner-Wunderlich syndrome. Arch Gynecol Obstet. 2014;290(5):947-50. https://doi.org/10.1007/ s00404-014-3286-5

15. Tong J, Zhu L, Lang J. Clinical characteristics of 70 patients with Herlyn-Werner-Wunderlich syndrome. Int J Gynaecol Obstet. 2013;121(2):173-5. https:// doi.org/10.1016/j.ijgo.2012.11.023

16. Yavuz A, Bora A, Kurdoğlu M, Goya C, Kurdoğlu Z, Beyazal M, et al. Herlyn-Werner-Wunderlich syndrome: merits of sonographic and magnetic resonance imaging for accurate diagnosis and patient management in 13 cases. J Pediatr Adolesc Gynecol. 2015;28(1):47-52. https://doi.org/10.1016/j.jpag.2014.03.004

17. Mohd I, Khan I, Cimona L. Herlyn-Werner-Wunderlich syndrome - a rare genitourinary anomaly in females: a series of four cases. Pol J Radiol 2018; 83(1):306-310. https://doi.org/10.5114/pjr.2018.77026

18. Gungor F, Dural O, Yasa C, Kirpinar G, Akhan S. Diagnosis, management, and outcome of obstructed hemivagina and ipsilateral renal agenesis (OHVIRA syndrome): Is there a correlation between MRI findings and outcome?. Clinical Imaging. 2020;59(2) 172178. https://doi.org/10.1016/j.clinimag.2019.11.013
19. Kapczuk K, Iwaniec K, Kedzia W. Obstructive Mullerian Anomalies in Menstruating Adolescent Girls: A Report of 22 Cases. J Pediatr Adolesc Gynecol. 2018; 31(3): 252-257. https://doi.org/10.1016/j. jpag.2017.09.013

20. Fascilla FD, Olivieri C, Cannone R, De Palma D, Manosperta F, Costantino AS, et al. In-office Hysteroscopic Treatment of Herlyn-Werner-Wunderlich Syndrome: A Case Series. J Minim Invasive Gynecol. 2020;27(7):1640-5. https://doi.org/10.1016/j. jmig.2020.04.013

21. Gholoum S, Puligandla PS, Hui T, Su W, Quiros E, Laberge J-M. Management and outcome of patients with combined vaginal septum, bifid uterus, and ipsilateral renal agenesis (Herlyn-Werner-Wunderlich syndrome). J Pediatr Surg. 2006;41(5):987-92. https://doi.org/10.1016/j.jpedsurg.2006.01.021

22. Mallmann MR, Reutter H, Mack-Detlefsen B, Gottschalk I, Geipel A, Berg C, et al. Prenatal Diagnosis of Hydro(metro)colpos: A Series of 20 Cases. Fetal Diagn Ther. 2019;45(1):62-8. https://doi. org/10.1159/000486781

23. Oestreich AE. Herlyn-Werner-Wunderlich syndrome: uterus didelphys, blind hemivagina and ipsilateral renal agenesis. Sonographic and MR findings in 11 cases. Yearbook of Diagnostic Radiology. 2008; 37(7) :130_ 1. https://doi.org/10.1016/S0098-1672(08)79140-9

24. Liu M, Zhang L, Xia Y, Huang X, Ye T, Zhang Y, et al. New Consideration of Herlyn-Werner-Wunderlich Syndrome Diagnosed by Ultrasound. J Ultrasound Med. 2021;40(9):1893-1900. https://doi. org/10.1002/jum.15572

25. Tong J, Zhu L, Chen N, Lang J. Endometriosis in association with Herlyn-Werner-Wunderlich syndrome. Fertil Steril. 2014;102(3):790-4. https://doi. org/10.1016/j.fertnstert.2014.05.025

26. Tuna T, Estevao-Costa J, Ramalho C, Fragoso AC. Herlyn-Werner-Wunderlich Syndrome: Report of a Prenatally Recognised Case and Review of the Literature, Pediatric Case Reports, Urology. 2018;125(2): 205-209. https://doi.org/10.1016/j. urology.2018.12.022

27. Nishu DS, Uddin MM, Akter K. Herlyn-WernerWunderlich syndrome presenting with dysmenorrhea: a case report Journal of Medical Case Reports. 2019; 13(2):323-326. https://doi.org/10.1186/s13256019-2258-6 
28. Fachin CG, Rocha JL, Maltoni AA, Lima RL, Zendim VA, Agulham MA, et al. Herlyn-Werner-Wunderlich syndrome: diagnosis and treatment of an atypical case and review of literature, International Journal of Surgery Case Reports. 2019; 63(1):129-134. https:// doi.org/10.1016/j.ijscr.2019.08.035

29. Mittal R.Herlyn-Werner-Wunderlich Syndrome, The Journal of Obstetrics and Gynecology of India. 2015; 66(2):128-130. https://doi.org/10.1007/s13224015-0707-8

30. Salastekar N, Coelho M, Majmudar A, Gupta S.Herlyn-Werner-Wunderlich syndrome: A rare cause of abdominal pain and dyspareunia. Radiology Case Reports. 2019; 14(1): 1297-1300. https://doi. org/10.1016/j.radcr.2019.08.003

31. Jung E, Cho M, Kim D, Byun J, Kim Y, Jeon D et al. Herlyn-Werner-Wunderlich syndrome An unusual presentation with pyocolpos. Obstet Gynecol Sci. 2017;60(4):374-377. https://doi.org/10.5468/ ogs.2017.60.4.374

32. Gupta N, Gandhi D, Gupta S, Goyal P, Li S, Kumar Y. A Variant of Herlyn-Werner-Wunderlich Syndrome Presenting With Acute Abdomen: A Case Report and Review of Literature. Global Pediatric Health. 201820 (5): 1-5. https://doi. org/10.1177/2333794X18769817

33. Jia G, Chai W, Cui M, Wen Y, Cui L, Gong F. A case report on Herlyn-Werner-Wunderlich syndrome with spontaneous abortion. Clinical Case Report. Medicine. 2018;97(36):1-4. https://doi.org/10.1097/ MD.0000000000012004

34. Cappello S, Piccolo, E., Cucinelli, F. Successful preterm pregnancy in a rare variation of Herlyn-WernerWunderlich syndrome: a case report BMC Pregnancy and Childbirth. 2018;18(1):498-502. https://doi. org/10.1186/s12884-018-2133-2

35. Reis M, Vicente A, Cominho J, Gomes A, Martins L, Nunes F. Pyometra and Pregnancy with HerlynWerner-Wunderlich Syndrome. Rev. Bras. Ginecol. Obstet.2016;38(12):623-628. http://dx.doi. org/10.1055/s-0036-1594304.

36. Miyazaki Y, Orisaka M, Nishino C, Onuma T, Kurokawa T, Yoshida Y. Herlyn-Werner-Wunderlich syndrome with cervical atresia complicated by ovarian endometrioma: A case report. J. Obstet. Gynaecol. Res. 2019;46(2):1-5. https://doi.org/10.1111/jog.14175
37. Angotti R, Molinaro F, Bulotta AL, Bindi E, Cerchia E, Sica M, et al. Herlyn-Werner-Wunderlich syndrome: An "early" onset case report and review of Literature International Journal of Surgery Case Reports. 2015; 11(2): 59-63. https://doi.org/10.1016/j. ijscr.2015.04.027

38. Vázquez JA, Sanz E, Vigil S, Aguado A. Síndrome de Herlyn-Werner-Wünderlich de diagnóstico neonatal. An Pediatr.2020; 94(4):263-264. https://doi. org/10.1016/j.anpedi.2020.06.005

39. Rastogi A, Khamesra A.Herlyn- Werner- Wunderlich Syndrome: A Rare Urogenital. Anomaly Masquerading as Acute Abdomen. Indian Journal of Pediatrics. 2010; 77(8):917. https://doi.org/10.1007/s12098010-0148-4

40. Tangshewinsirikul C, Dulyaphat W, Diawtipsukon S, Tingthanatikul Y, Satirapod C, Wattanasirichaigoon D. Successful pregnancy outcome in Herlyn-Werner-Wunderlich syndrome with pyocolpos: A case report and literature review. Clinical case reports. 2020; 8(11): 2217-2222. https://doi.org/10.1002/ ccr3.3080

41. Gungor F, Bastu E, Gulsen G, Kurek M, Engin S. OHVIRA syndrome presenting with acute abdomen: a case report and review of the literature. Clinical Imaging. 2014; 38(2): 357-359. https://doi.org/10.1016/j. clinimag.2013.12.011

42. Yakıştıran B, Şükür YE, Turgay B, Atabekoğlu C. True management of Obstructed Hemi-vagina and Ipsilateral Renal Anomaly syndrome. Turk J Obstet Gynecol 2016;13(4):208-11. https://doi.org/10.4274/ tjod. 23434

43. Takagi K, Matsunami K, Imai A. Uterovaginal duplication with blind hemivagina and ipsilateral renal agenesis: Review of unusual presentation. Journal of Obstetrics and Gynaecology, May 2010; 30(4): 350353. https://doi.org/10.3109/01443611003763416

44. Sen KK, Balasubramaniam D, Kanagaraj V. Magnetic resonance imaging in obstructive müllerian anomalies. Journal of Human Reproductive Sciences. 2013; 6(2):162-164. https://doi.org/10.4103/09741208.117167

45. Mandava A, Prabhakar, R, Smitha S. OHVIRA Syndrome (obstructed hemivagina and ipsilateral renal anomaly) with Uterus Didelphys, an Unusual Presentation J Pediatr Adolesc Gynecol. 2012; 25 (2):23-25. https://doi.org/10.1016/j.jpag.2011.11.004 
46. Bajaj SK, Misra R, Thukral BB, Gupta R. OHVIRA: Uterus didelphys, blind hemivagina and ipsilateral renal agenesis: Advantage MRI. Journal of Human Reproductive Sciences. 2012; 5(1):67-70. https:// doi.org/10.4103/0974-1208.97811

47. Jindal G., Kachhawa, S., Meena, G., \& Dhakar, G.Uterus didelphys with unilateral obstructed hemivagina with hematometrocolpos and hematosalpinx with ipsilateral renal agenesis.J Hum Reprod Sci. 2009; 2(2):87-89. https://doi.org/10.4103/09741208.57230

48. Vivier PH, Liard A, Beurdeley M. Uterus didelphys, hemihydrocolpos and empty ipsilateral lumbar fossa in a newborn girl: involuted dysplastic kidney rather than renal agenesis. Pediatr Radiol. 2011;41(1):12051207. https://doi.org/10.1007/s00247-011-2046-y

49. Mishra N, Ng S.Sonographic diagnosis of Obstructed Hemivagina and Ipsilateral Renal Anomaly Syndrome: a report of two cases. AJUM November 2014;17(4):153158. https://doi.org/10.1002/j.2205-0140.2014. tb00238.x

50. Wu T-H, Wu T-T, Ng Y-Y, Ng S-C, Su P-H, Chen J-Y, et al. Herlyn-Werner-Wunderlich Syndrome Consisting of Uterine Didelphys, Obstructed Hemivagina and Ipsilateral Renal Agenesis in a Newborn. 2012; 53 (2): 68-71. https://doi.org/10.1016/j. pedneo.2011.11.014

51. Cozzolino M, Corioni S, Magro Malosso ER, Sorbi F, Mecacci F. Two successful pregnancies in HerlynWerner-Wunderlich syndrome. J Obstet Gynaecol. 2014;34(6):534-5. https://doi.org/10.3109/01443 615.2014 .914898

52. Sanghvi Y, Shastri P, Mane SB, Dhende NP. Prepubertal presentation of Herlyn-Werner-Wunderlich syndrome: a case report. J Pediatr Surg. 2011;46(6):1277-80. https://doi.org/10.1016/j.jpedsurg.2011.02.067

53. Pereira N, Anderson SH, Verrecchio ES, Brown M, Glassner MJ. Hemivaginal Septum Resection in a Patient With a Rare Variant of Herlyn-WernerWunderlich Syndrome. Journal of Minimally Invasive Gynecology. 2014; 21(1): 1113-7. https://doi. org/10.1016/j.jmig.2014.05.001

54. Han BH, Park SB, Lee YJ, Lee KS, Lee YK. Uterus didelphys with blind hemivagina and ipsilateral renal agenesis (Herlyn-Werner-Wunderlich syndrome) suspected on the presence of hydrocolpos on prenatal sonography. J Clin Ultrasound. 2013;41(6):380-2. https://doi.org/10.1002/jcu.21950

55. Wozniakowska E, Torres A, Milart P, Wozniak S, Czuczwar P, Szkodziak P, et al. Delayed diagnosis of Herlyn-Werner-Wunderlich syndrome due to microperforation and pyocolpos in obstructed vaginal canal. J Pediatr Adolesc Gynecol. 2014;27(4):79-81. https:// doi.org/10.1016/j.jpag.2013.07.009

56. Dorais J, Milroy C, Hammoud A, Chaudhari A, Gurtcheff S, Peterson CM. Conservative treatment of a Herlyn-Werner-Wunderlich müllerian anomaly variant, noncommunicating hemiuterus with Gartner duct pseudocyst. J Minim Invasive Gynecol. 2011;18(2):262-6. https://doi.org/10.1016/j. jmig.2010.12.006

57. Watanabe Y, Etoh T, Nakai H. Adenocarcinoma of the lower female genital tract in patients with Herlyn-Werner-Wunderlich syndrome. Am J Obstet Gynecol. 2012;207(6):5-6. https://doi.org/10.1016/j. ajog.2012.09.009

58. Liang H-I, Fu S-C, Yin C-H, Chang C-C. HerlynWerner-Wünderlich syndrome: An unusual case with presentation of menorrhagia. Taiwan J Obstet Gynecol. 2020;59(6):948-51. https://doi.org/10.1016/j. tjog.2020.09.026

59. Nabeshima H, Nishimoto M, Shiga N, Utsunomiya H, Yaegashi N. Laparoscopic Strassman Metroplasty in a Postmenarcheal Adolescent Girl With Herlyn-WernerWunderlich Müllerian Anomaly Variant, Obstructed Noncommunicating Didelphic Uterus Without Gartner Duct Pseudocyst. Journal of Minimally Invasive Gynecology. 2013; 20 (1): 255-8. https://doi. org/10.1016/j.jmig.2012.10.016

60. Asha B, Manila K. An unusual presentation of uterus didelphys with obstructed hemivagina with ipsilateral renal agenesis. Fertil Steril. 2008;90(3):849-851. https://doi.org/10.1016/j.fertnstert.2007.08.003

61. Roth M, Mingin G, Dharamsi N, Psooy K, Koyle M. Endoscopic ablation of longitudinal vaginal septa in prepubertal girls: a minimally invasive alternative to open resection. J Pediatr Urol. 2010 Oct;6(5):464-8. https://doi.org/10.1016/j.jpurol.2009.12.009

62. Rana R, Pasrija S, Puri M. Herlyn-Werner-Wunderlich syndrome with pregnancy: a rare presentation. Congenit Anom . 2008;48(3):142-3. https://doi. org/10.1111/j.1741-4520.2008.00195.x 
63. Kumar S, Rajagopal KV, Karegowda LH, Chaitanya RK. Herlyn-Werner-Wunderlich syndrome: a rare cause of acute urinary retention in an adolescent girl.Case Reports. 2015;20(9):63-68. https://doi.org/10.1136/ bcr-2015-209638

64. Güdücü N, Gönenç G, Iş̧̧i H, Yiğiter AB, Dünder I. Herlyn-Werner-Wunderlich syndrome--timely diagnosis is important to preserve fertility. J Pediatr Adolesc Gynecol. 2012;25(5):e111-2. https://doi. org/10.1016/j.jpag.2012.05.013

65. Sarac A, Demir MK. Herlyn-Werner-Wunderlich syndrome: a rare cause of infertility (2009: 2b). Eur Radiol. 2009;19(5):1306-8. https://doi.org/10.1007/ s00330-008-1085-8

66. Noviello C, Romano M, Nino F, Martino A, Cobellis $\mathrm{G}$. Clinical and radiological findings for early diagnosis of Herlyn-Werner-Wunderlich syndrome in pediatric age: experience of a single center. Gynecological Endocrinology. 2018; 34 (2): 56-8. https://doi.org/1 $0.1080 / 09513590.2017 .1332178$

67. Yung SF, Ngu S-F, Cheung VT. Late presentation of a variant of Herlyn-Werner-Wunderlich syndrome. Int J Gynaecol Obstet. 2016;133(2):238-9. https://doi. org/10.1016/j.ijgo.2015.10.006

68. Aveiro AC, Miranda V, Cabral AJ, Nunes S, Paulo F, Freitas C. Herlyn-Werner-Wunderlich syndrome: a rare cause of pelvic pain in adolescent girls. BMJ Case Rep.2011(4):42-47. https://doi.org/10.1136/ bcr.04.2011.4147

69. Beer WM, Carstairs SD. Herlyn Werner Wunderlich syndrome: an unusual presentation of acute vaginal pain. J Emerg Med. 2013;45(4):541-3. https://doi. org/10.1016/j.jemermed.2013.03.035

70. Schutt AK, Barrett MR, Trotta BM, Stovall DW. Perioperative evaluation in Herlyn-Werner-Wunderlich syndrome. Obstet Gynecol. 2012;120(4):948-51. https://doi.org/10.1097/AOG.0b013e318265e35a

71. Del Vescovo R, Battisti S, Di Paola V, Piccolo CL, Cazzato RL, Sansoni I, et al. Herlyn-Werner-Wunderlich syndrome: MRI findings, radiological guide (two cases and literature review), and differential diagnosis. BMC Med Imaging. 2012 9;12:4. https://doi. org/10.1186/1471-2342-12-4

72. Cox D, Ching BH. Herlyn-Werner-Wunderlich syndrome: a rare presentation with pyocolpos. J Radiol Case Rep. 2012;6(3):9-15. https://doi.org/10.3941/ jrcr.v6i3.877
73. Dogan A, Uyar I, Demirtas GS, Ekin A, Gulhan I, Ertas IE, et al. Urinary Incontinence in Puberty: A Rare Clinical Presentation of the Herlyn-WernerWunderlich Syndrome. J Pediatr Adolesc Gynecol. 2016;29(6):e101-3. https://doi.org/10.1016/j. jpag.2016.07.013

74. Karaca L, Pirimoglu B, Bayraktutan U, Ogul H, Oral A, Kantarci M. Herlyn-Werner-Wunderlich Syndrome: A Very Rare Urogenital Anomaly in a Teenage Girl.The Journal of Emergency Medicine. 2015;48 (2):73-5. https://doi.org/10.1016/j.jemermed.2014.09.064

75. Moshiri M, Seyal AR, Cruite I, Bhargava P. HerlynWerner-Wunderlich syndrome with a partially obstructed hemivagina. Radiol Case Rep. 2012;7(4):800. https://doi.org/10.2484/rcr.v7i4.800

76. Khaladkar SM, Kamal V, Kamal A, Kondapavuluri SK. The Herlyn-Werner-Wunderlich Syndrome - A Case Report with Radiological Review.Polish Journal of Radiology. 2016; 81(1): 395-400. https://doi. org/10.12659/PJR.897228

77. Kozłowski M, Nowak K, Boboryko D, Kwiatkowski S, Cymbaluk-Płoska A. Herlyn-Werner-Wunderlich Syndrome: Comparison of Two Cases. Int J Environ Res Public Health. 2020;17(19). https://doi.org/10.3390/ ijerph17197173

78. Ellspermann R, Sirhari C, Chapin E, Nelson M. Pointof-care Ultrasound Aiding in the Diagnosis of HerlynWerner-Wunderlich Syndrome. Clinical Practice and Cases in Emergency Medicine. 2017; 1(2): 370-3. https://doi.org/10.5811/cpcem.2017.7.34089

79. Hamidi H, Haidary N. Late presentation, MR imaging features and surgical treatment of Herlyn-WernerWunderlich syndrome (classification 2.2); a case report. BMC Women's Health. 2018; 18 (1):161-165 https://doi.org/10.1186/s12905-018-0655-4

80. Meneses AD, Filho WM, Raulino DM, Martins EB, Vieira SC. Herlyn-Werner-Wunderlich Syndrome with Ureteric Remnant Abscess Managed Laparoscopically: A Case Report. Oman Med J. 2017 Mar;32(2):15760. https://doi.org/10.5001/omj.2017.28

81. Widyakusuma LS, Lisnawati Y, Pudyastuti S, Haloho AH. A rare case of pelvic pain caused by HerlynWerner-Wunderlich Syndrome in an adult: A case report. International Journal of Surgery Case Reports. 2018; 49 (2): 106-9. https://doi.org/10.1016/j. ijscr.2018.06.022 
82. Tan YG, Laksmi NK, Yap T-L, Sadhana N, Ong CC. Preventing the $\mathrm{O}$ in OHVIRA (Obstructed Hemivagina Ipsilateral Renal Agenesis): Early Diagnosis and Management of Asymptomatic Herlyn-WernerWunderlich Syndrome. Journal of Pediatric Surgery. 2020; 55(2):1377-80. https://doi.org/10.1016/j. jpedsurg.2019.06.006

\section{FINANCIACIÓN}

Los autores declaran que no recibieron recursos financieros para la realización de este trabajo, tampoco becas, subvenciones, beneficios, ni pagos.

\section{CONTRIBUCIÓN DE LOS AUTORES}

Oscar Octalivar Gutiérrez Montufar: elaboración del documento desde su concepción y diseño hasta la adquisición de la información, revisión del contenido intelectual, elaboración de fotos y participación en el diseño del material gráfico.
Claudia Patricia Zambrano Moncayo: revisión del contenido intelectual y aprobación de la versión enviada a proceso editorial.

Maria Camila Otálora Gallego: revisión del contenido intelectual y aprobación de la versión enviada a proceso editorial.

Angy Lorena Meneses Parra: elaboración del documento desde su concepción, diseño hasta la adquisición de la información y revisión del contenido intelectual.

Ivonne Díaz Yamal: revisión del contenido intelectual y aprobación de la versión enviada a proceso editorial. 